\title{
The No Core Gamow Shell Model for ab-initio Nuclear Structure Calculations
}

\author{
G. Papadimitriou ${ }^{1}$, B.R. Barrett ${ }^{1}$, J. Rotureau ${ }^{3}$, N. Michel $^{2}$, and M. Płoszajczak ${ }^{4}$ \\ ${ }^{1}$ Department of Physics, University of Arizona, Tucson, AZ 85721, USA \\ ${ }^{2}$ Department of Physics and Astronomy, University of Tennessee, Knoxville, Tennessee 37996, USA \\ ${ }^{3}$ Fundamendal Physics, Chalmers University of Technology, 41296 Göteborg, Sweden \\ ${ }^{4}$ Grand Accélérateur National d'lons Lourds (GANIL), CEA/DSM-CNRS/IN2P3, BP 55027, F-14076 Caen \\ Cedex 5, France
}

\begin{abstract}
We apply the Berggren basis in a No-Core Shell Model framework to calculate ground state (g.s.) energies of ${ }^{3} \mathrm{H},{ }^{4} \mathrm{He}$ and ${ }^{5} \mathrm{He}$. In our studies we use the Argonne $v_{18}$ and the chiral $\mathrm{N}^{3} \mathrm{LO}$ potentials, both of which are renormalized via a $\mathrm{V}_{\text {low-k }}$ process.
\end{abstract}

\section{Introduction}

One of the methods for calculating nuclear properties from first principles is the No-Core Shell Model (NCSM) [1]. Even though this method was applied with much success in the description of wellbound states, some problems arose when it was used for calculating properties of nuclei with a large radial extent ("halo" nuclei) or in the description of unbound states. The basic source of the problem was that a very large number of $\mathrm{HO}$ states are required to capture the physics of weakly bound "halo" nuclei, due to the Gaussian asymptotic behavior of the $\mathrm{HO}$ wavefunction. This does not mean that using HO states is wrong, but that they do not provide the correct scheme to calculate weakly-bound and unbound systems. This usually leads to non-converged results and the extrapolations to "infinite" basis were not very successful, until recently [2,3]. Additionally, it was soon realized that the coupling to the continuum can also have an appreciable effect on the properties of states close to threshold. This leads to a modification of many-body methods in order to include the continuum coupling in their frameworks [4-8]. In this work, our method of choice is the No-Core Gamow Shell Model, where the continuum is taken into account via the diagonalization of the many-body Hamiltonian in the Berggren basis [9].

\section{The No-Core Gamow Shell Model.}

Inspired by the success of the Gamow Shell Model (GSM) [8], we will apply Berggren's basis in a No-Core framework. The Hamiltonian we are considering has the form:

$$
H=\sum_{i<j}^{A} \frac{\left(\vec{p}_{i}-\vec{p}_{j}\right)^{2}}{2 m A}+\sum_{i<j}^{A} V_{i j}^{N N}
$$

This is an Open Access article distributed under the terms of the Creative Commons Attribution License 2.0, which permits unrestricted use, distribution, and reproduction in any medium, provided the original work is properly cited. 
where $A$ is the number of nucleons and $V_{i j}^{N N}$ a realistic two-body interaction. The basis will consist of resonant states (bound states and resonances) and also non-resonant continuum states, which are distributed along a contour $\mathcal{L}$ below the resonant states. It was shown by Berggren [10] that this set is complete via the completeness relation:

$$
\sum_{\text {res }}\left|u_{\text {res }}\right\rangle\left\langle\widetilde{u_{\text {res }}}\left|+\int_{\mathcal{L}}\right| u_{k}\right\rangle\left\langle\widetilde{u_{k}}\right| d k=1
$$

where the tilde symbols mean that the complex conjugation has an impact only on the angular part of the basis functions, and $k$ is the linear momentum associated with the energy through the relation $k=\sqrt{\frac{2 m E}{\hbar}}$ and $E$ is, in general, complex. The basis states correspond to solutions of the single particle (s.p.) Schrödinger equation with outgoing boundary conditions (resonant part) or scattering boundary conditions (non-resonant part) and for a potential that has a finite depth and, thus, can support such solutions (Woods-Saxon, Hartree-Fock, etc.). In numerical applications, the integral in (2) is discretized and we obtain a discrete set of basis states:

$$
\sum_{\text {res }}\left|u_{\text {res }}\right\rangle\left\langle\widetilde{u_{r e s}}\left|+\sum_{i}\right| u_{k i}\right\rangle\left\langle\widetilde{u_{k i}}\right| \omega_{i}=1
$$

where $\omega_{i}$ stands for the weight associated with the quadrature used (Gauss-Legendre in our case). Additionally, we truncate the s.p. space up to a momentum $k_{\max }$. Results, for a sufficient number of basis states, are, in general, independent of the $k_{\max }$ value. After obtaining the s.p. basis, we construct many-body Slater determinant basis states, upon which the Hamiltonian (1) is diagonalized. The Hamiltonian matrix in our scheme is, in general, complex symmetric with complex eigenvalues. For the diagonalization of the non-Hermitian matrix we can use conventional Hermitian matrix diagonalization techniques, such as Lanczos or Davidson, but generalized to the non-Hermitian case [8]. Additionally, we also use the technique of the Density Matrix Renormalization Group (DMRG) for the solution of the large eigenvalue problem $[9,11]$. In this approach the system is growing iteratively by adding non-resonant shells one-by-one, and the most important states are selected as the ones that correspond to the maximum eigenvalues of the density matrix.

\section{Results and Discussion}

\section{$3.1{ }^{3} \mathrm{H}$ and ${ }^{4} \mathrm{He}$}

We applied the NCGSM to the bound nuclei ${ }^{3} \mathrm{H}$ and ${ }^{4} \mathrm{He}$. In spite of the fact that we would, in general, use two-body matrix elements (TBMEs) of the interactions in the Berggren basis, in practice we use TBMEs of interactions in a $\mathrm{HO}$ basis. We, hence, take advantage of the unique property of the $\mathrm{HO}$ basis, which provides a transformation in a closed form, from the relative coordinates to the laboratory coordinates, in which our wavefunctions are expressed. In particular, we approximate TBMEs in the Berggren basis by (see [9] and references therein):

$$
\left\langle a b\left|V_{N N}\right| c d\right\rangle=\sum_{\alpha \beta \gamma \delta}^{N_{\max }}\langle a b \mid \alpha \beta\rangle\left\langle\alpha \beta\left|V_{N N}\right| \gamma \delta\right\rangle\langle\gamma \delta \mid c d\rangle,
$$

so that we end up calculating only overlaps between Berggren states (Latin letters) and HO states (Greek letters). In all the current calculations we took $\mathrm{n}_{\max }=10$ for the HO states $(\mathrm{N}=2 \mathrm{n}+\ell)$ and the $\mathrm{HO}$ length parameter was $b=1.5 \mathrm{fm}$. Only the interaction was treated in this approximation. For the triton the basis was generated via a Gamow Hartree Fock (GHF) calculation [9, 12]. We employ the $\mathrm{V}_{\text {low-k }}$ [13] Argonne $v_{18}$ potential [14] ${ }^{1}$. Additionally, we include the real $\mathrm{s}_{1 / 2}, \mathrm{p}_{3 / 2}$ and $\mathrm{p}_{1 / 2}$ continua

\footnotetext{
${ }^{1}$ In all the following when we mention $\mathrm{V}_{\text {low- } k}$ we will mean a $\Lambda=1.9 \mathrm{fm}^{-1} \mathrm{~V}_{\text {low-k }}$
} 
along the real $k$-axis, which extends up to a $k_{\max }=4 \mathrm{fm}^{-1}$. For each partial wave (both protons and neutrons) we consider 18 scattering shells and the remaining basis sets, up to g-waves, are taken as HO states with $b=1.5 \mathrm{fm}$. It is apparent that we do not use complex states for the description of the triton (and ${ }^{4} \mathrm{He}$ later on). The matrix is real and symmetric with real eigenvalues. The basis then for the s-p states satisfies Newton's completeness [15], which includes bound states and scattering states on the real-axis. The purpose of this calculation is purely numerical, in order to test the convergence of our basis against exact bound states methods. The test was successful and we report that we obtain the value of $-8.39 \mathrm{MeV}$ [9], whereas the Faddeev calculation is $-8.47 \mathrm{MeV}$ [16].

For ${ }^{4} \mathrm{He}$ we are using the same space but with a $\mathrm{V}_{l o w-k} \mathrm{~N}^{3} \mathrm{LO}$ interaction [17]. In this system we are performing a DMRG diagonalization. The states that we keep each time we add one scattering shell $\left(\mathrm{N}_{\text {step }}\right)$, is controlled by the parameter $\epsilon$, which is expressed as:

$$
\left|1-\operatorname{Re}\left(\sum_{i=1}^{N} \rho_{i}\right)\right|<\epsilon,
$$

where $\rho_{i}$ stands for the eigenvalues of the density matrix. The smaller the $\epsilon$, the more states of the density matrix we keep. In this application we used a parameter $\epsilon=10^{-6}$, which resulted in keeping approximately $\mathrm{N} \sim 180$ states of the density matrix. The maximum dimension of the Hamiltonian matrix to diagonalize was then about 6000 . The converged result at the end of the DMRG iteration is $-27.48 \mathrm{MeV}[9]$.

\section{$3.2{ }^{5} \mathrm{He}$}

Next we apply the NCGSM to calculate the resonance parameters of the ${ }^{5} \mathrm{He}$ g.s., using the $\mathrm{V}_{\text {low }-k}$ $\mathrm{N}^{3} \mathrm{LO}$ potential. In our formalism the resonance parameters will be identified as the eigenvalues of the complex-symmetric Hamiltonian matrix. The position of the resonance will then be the real part of the energy, while the imaginary part is related to the width by the formula: $\Gamma=-2 \operatorname{Im}(\mathrm{E})$. An advantage of using Berggren's states is that the complex eigenvalues $(\mathrm{E})$ are the ones, which correspond to states with purely outgoing-wave solutions. This is the case for the particle unbound ${ }^{5} \mathrm{He}$ nucleus. For our calculation we use a complex Berggren basis, which includes the complex $0 \mathrm{p}_{3 / 2}$ s.p. resonance and the associated complex continua, which are distributed along the complex contour $\mathcal{L}$. The complex $\mathrm{p}_{3 / 2}$ contour is taken in such way, so as to include the s.p. resonance and it was discretized with 18 points. The remaining $\left(\mathrm{s}_{1 / 2}\right.$ and $\left.\mathrm{p}_{1 / 2}\right)$ scattering states are taken along the real momentum axis, whereas states with $\ell>2$ are taken as HO states. In this space the Hamiltonian matrix is complex symmetric, and we will apply the DMRG method to obtain the eigensolutions. Our converged result for a DMRG truncation parameter $\epsilon=10^{-6}$ gave $\operatorname{Re}\left(\mathrm{E}_{5_{\mathrm{He}}}\right)=-26.31 \mathrm{MeV}$ and $\Gamma=-2 \operatorname{Im}\left(\mathrm{E}_{5_{\mathrm{He}}}\right)=400$ $\mathrm{keV}$. We obtain then the position of the resonance at an energy $1.17 \mathrm{MeV}$ above the $\alpha+\mathrm{n}$ threshold with a width $\Gamma=400 \mathrm{keV}$ [9]. We will compare this result with the value extracted from experiment. Experimentally, resonance parameters can be extracted in two ways [18]. First, one could apply the conventional R-matrix approach on the real axis and use the Lane and Thomas prescription for the extraction of positions and widths of a state [19]. The second way is the "extended" R-matrix approach [20,21], which associates the resonance parameters with the complex poles of the S-matrix. According to G.M. Hale [22], this is the recommended prescription, and it is also the most appropriate for comparison to the NCGSM results, since our complex eigenvalues correspond, indeed, to the poles of the S-matrix with the correct asymptotic behavior. The "extended" R-matrix approach gives the position of the $\left(\frac{3}{2}\right)^{-}$state at an energy $798 \mathrm{keV}$ above the $\alpha+\mathrm{n}$ threshold and with a width $\Gamma=$ $648 \mathrm{keV}$. The difference we observe between our results and the experimental results is attributed to 
the specific interaction that we employ and/or to missing many-body forces. We know already that the position of such unbound states, which have a non Breit-Wigner character (broad states), heavily depends on the specific characteristics of the NN (or NNN) interaction. This was shown in the manybody frameworks of the NCSM merged with the Resonating Group Method [4, 23, 24] and also in the Green's Function Monte Carlo approach [6]. It needs to be mentioned that, because of the broad nature of the ${ }^{5} \mathrm{He}$ g.s., results would also depend on the method used to extract such a state from the experimental data. We find that if one uses the conventional R-matrix approach on the real axis the position of the g.s. is at $0.963 \mathrm{MeV}$ above the $\alpha+\mathrm{n}$ threshold with a width $\Gamma=985 \mathrm{MeV}$ and both values are different by about $200 \mathrm{keV}$ as compared to the "extended" R-matrix estimations.

In conclusion, we applied the NCGSM to calculate g.s. energies of ${ }^{3} \mathrm{H}$ and ${ }^{4} \mathrm{He}$ to benchmark

our algorithms. We also applied our technique to calculate the resonance parameters of the ${ }^{5} \mathrm{He}$ g.s. from first principles. The resonance characteristics are obtained naturally in the NCGSM, as eigenvalues of the Hamiltonian matrix, and the correct outgoing behavior is also preserved. The result is satisfactory, as compared with experiment and taking into consideration the difficulties involved with the calculation of such broad states.

\section{References}

[1] B. R. Barrett, P. Navrátil and J. P. Vary, Prog. Part. Nucl. Phys. 69, 131 (2013)

[2] S. A. Coon, et al, Phys. Rev. C 86, 054002 (2012)

[3] S. N. More, et al, Phys. Rev. C 87, 044326 (2013)

[4] S. Quaglioni, and P. Navrátil, Phys. Rev. C 79, 044606 (2009)

[5] S. Baroni, P. Navrátil, and S. Quaglioni, Phys. Rev. Lett. 110, 022505 (2013)

[6] K. M. Nollett, et al, Phys. Rev. Lett. 99, 022502 (2007)

[7] G. Hagen, D.J. Dean, M. Hjorth-Jensen and T. Papenbrock, Phys. Lett. B 656, 169 (2007)

[8] N. Michel, et al, J. Phys. G: Nucl. Part. Phys. 36, 013101 (2009)

[9] G. Papadimitriou, et al, Phys. Rev. C 88, 044318 (2013)

[10] T. Berggren, Nucl. Phys. A 109, 265 (1968)

[11] J. Rotureau, et al, Phys. Rev. Lett 97, 110603 (2006); Phys. Rev. C 79, 114304 (2009)

[12] N. Michel, Eur. Phys. J. A. 73, 523 (2009)

[13] S. K. Bogner, T.T.S. Kuo and A. Schwenk, Phys. Rep. 386, 1 (2003)

[14] R. B. Wiringa, V. G. J. Stoks, and R. Schiaville, Phys. Rev. C 51, 38 (1995)

[15] R.G. Newton, J.Math.Phys. 1, 319 (1960)

[16] A. Nogga, S.K.Bogner, and A. Schwenk, Phys. Rev. C 70, 061002(R) (2004)

[17] D. R. Entem and R. Machleidt, Phys. Rev. C 68, 041001(R) (2003)

[18] D. R. Tilley, et al, Nucl. Phys. A 708, 3 (2002)

[19] A. M. Lane and R. G. Thomas, Rev. Mod. Phys. 30, 257 (1958)

[20] G. M. Hale, R. E. Brown and N. Jarmie, Phys. Rev. Lett. 59, 763 (1987)

[21] A. Csótó and G. M. Hale, Phys. Rev. C 55, 536 (1997)

[22] G. M. Hale, private communication

[23] S. Quaglioni, and P. Navrátil, Phys. Rev. Lett. 101, 092501 (2008)

[24] P. Navrátil, R. Roth and S. Quaglioni, Phys. Rev. C 82, 034609 (2010) 